\title{
Management of cystic pancreatic lesions found incidentally
}

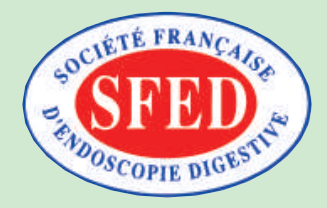

Author

Institution
M. Barthet, B. Napoléon, L. Palazzo, M. Chemali, J.-C. Letard, R. Laugier, J.-P. Arpurt, J. Boyer, C. Boustière, J.-M. Canard, J. Cassigneul, P.-A. Dalbiès, J. Escourrou, G. Gay, T. Ponchon, B. Richard-Molard, D. Sautereau, G. Tucat, B. Vedrenne

SFED Paris, France
Bibliography

Dol 10.1055/s-2007-966786

Endoscopy 2007; 39:

926-928 @ Georg Thieme

Verlag KG Stuttgart · New York ISSN 0013-726X

Corresponding author

T. Ponchon

SFED, 96 boulevard du

Montparnasse, 75014 Paris

France

Fax: +33140540066

secretariat@sfed.org
Solid and cystic pancreatic lesions are increasingly being found incidentally, owing to improved pancreatic imaging techniques, with ultrasonography, multidetector computed tomography (CT) scanning and magnetic resonance imaging (MRI), and a better knowledge of the characteristics and incidence of these lesions. One in every 5000 screening ultrasonographies reveals a potentially malignant cystic tumor of the pancreas [1]. Similarly to intraductal papillary mucin-producing tumors (IPMTs), more than $10 \%$ of pancreatic cystic tumors are identified incidentally [2]. Malignant cystic tumors are frequently symptomatic and it is therefore highly probable that a cystic tumor which is discovered accidentally will be benign $[2,3]$. The proportion of cystic tumors that are incidentally identified is increasing in France and the USA, rising from $8.8 \%$ in the 1980 s to $11.9 \%$ in the 1990 s [2].

\section{Diagnostic approach}

First it must be confirmed that the lesion is a cystic tumor, then the malignant or potentially malignant nature of the lesion must be assessed, and finally, if possible, the histological nature of the lesion should be defined.

To accomplish this, clinicians have, besides ultrasonography, three key complementary modalities at their disposal: the multidetector CT scanner, magnetic resonance imaging of the pancreas (MRP), and endoscopic ultrasound (EUS) that can be coupled with fine-needle aspiration (for cytology and markers). The combination of these three imaging techniques with a clinical evaluation of the patient is essential in any approach to diagnosing pancreatic cystic tumors $[3,4]$. The risk of potential or immediate malignant transformation of the pancreatic cystic lesion justifies this thorough and systematic check-up, even though it does not usually need to be carried out urgently [4].

\section{Diagnostic elements}

$\nabla$

There should be systematic evaluation of the following:

- Condition of the adjacent and distant pancreatic parenchyma

- Condition of the pancreatic duct (dilatation, communication with the cyst cavity)

- Size of the lesion

- Location of the lesion

- Unique or multifocal nature

- Size of the cysts in the case of multifocal lesions (size of the smallest and largest cysts)

- Presence of septa within the cystic cavity.

- Existence of central or peripheral calcification.

- Existence of endocystic polyps or nodules.

These elements contribute to the diagnosis of cystic tumor, the differential diagnosis, and the diagnosis of a malignant transformation.

Ultrasonography usually allows the assessment of the precise location, the size, the unique or multifocal nature of the lesion, the condition of the adjacent pancreatic parenchyma, and the size of the main pancreatic duct. The liquid nature of the lesion is shown by its anechoic pattern and the posterior reinforcement of the ultrasound beam transmission.

Multidetector CT scanning clearly identifies the anatomic location of the lesion, its anatomical connections and the presence of vascular enhancement. By these means, a recent French study obtained $100 \%$ specificity in diagnosing cystadenosarcoma on the basis of three radiological criteria (lobulation, cephalic location and absence of vascular enhancement) [5]. 
MRP usually allows the identification of ductal involvement (communication between the cyst cavity and the pancreatic ducts), the spread of cystic lesions into the pancreatic gland, and is therefore of particular relevance in diagnosing IPMT.

Endoscopic ultrasonography not only provides a clear study of the cystic wall, the presence of endocystic polyps or nodules, the condition of the adjacent pancreatic parenchyma and the presence of any microcysts [6], but can also be used for fine-needle aspiration of the cyst to obtain a cytological evaluation and assessment of molecular markers (such as CEA, CA19-9, amylase, and lipase).

\section{Positive diagnosis and diagnosis of malignancy}

It is not always easy to make a positive diagnosis of cystic tumors. There are two differential diagnoses, the first being pancreatic pseudocyst [7]. It is therefore essential to investigate the clinical background (alcohol abuse, history of pancreatitis, abdominal trauma) and to check whether the pancreatic parenchyma surrounding the lesion is normal; the best way of determining this is by EUS. False-positive results indicating pancreatic cystadenocarcinoma or degenerative IPMT have been described in the case of necrotic pseudocysts, but this erroneous diagnosis has occurred less often in recent years [2,7].

The other differential diagnosis is of pancreatic cystic endocrine tumor; these comprise about $2 \%$ of pancreatic cystic tumors $[4,7]$. These are often smaller lesions with walls of varying thickness or with a solid component. In over $50 \%$ of cases these tumors are not secreting, and the diagnosis can be tricky. Intense peripheral arterial enhancement is suggestive for this diagnosis.

The malignancy of a cystic lesion of the pancreas is first investigated by exploring the adjacent pancreatic parenchyma for a possible local invasion, particularly when there is a solid component within the cyst. The presence of a thick and irregular cystic wall, intracystic polyps or nodules, and peripheral rim calcification are predictive of malignancy [4,7-9]. EUS is without doubt the examination that provides the most accurate evaluation of malignancy,. EUS-guided fine-needle aspiration allows a cytology specimen to be taken by "scratching" the wall with the needle or by taking a sample of the polyp or nodule $[3,6]$. The findings from this cytological examination are often difficult to analyze and thus it needs to be performed by an experienced pathologist. The sample must be managed using conventional methods on slides and in a liquid medium using the monolayer spreading method.

\section{Diagnosis of etiology: the nature of the cyst and classification of the lesions) \\ $\nabla$}

Imaging data allow classification of the lesions according to the presence of communication with the pancreatic ducts (IPMT), the micro and/or macrocystic nature (cysts $\leq 2 \mathrm{~mm}$ are indicative of serous cystadenoma), and the presence of septae (mucinous cystadenoma).

Lesions are also classified according to the malignancy risk:

- Main duct IPMT. This has a high potential for malignant transformation (>30\%) requiring surgical resection.

- Branch duct IPMT. The potential for malignant transformation is average (10\% to $15 \%$ ), and multidisciplinary evaluation is needed before the decision on management. Communication between the cystic cavity and the pancreatic duct proves that this lesion is an IPMT. The issue is then to rule out signs predictive of malignant transformation (size greater than $3 \mathrm{~cm}$, the presence of intracystic polyps or nodules, or the presence of wall irregularities) and involvement of the main pancreatic duct [9].

- Mucinous cystadenoma. This also has a high potential for malignant transformation (>30\%) requiring surgical resection. This cystic lesion typically occurs in middle-aged women and is located in the body or tail of the pancreas. It presents as a mass lesion composed of one or more macrocystic spaces. In contrast to serous cystadenoma, mucinous cystadenoma has a distinct feature on CT imaging of vascular enhancement of the cystic wall [3-5]. The presence of rim calcification is predictive of malignancy [4].

- Solid pseudopapillary tumor. This has borderline potential for malignant transformation, requiring a surgical resection as it is locally aggressive and has a risk of metastasis. This type of tumor is the least common of the pancreatic cystic neoplasms $(<5 \%)$ and usually occurs in young women. When it is small in size, it can take on the characteristics of an endocrine tumor or resemble a combination of a solid and cystic tumor with, at most, a honeycomb-like appearance. When it is large, it may be altered through necrosis or hemorrhage [2].

- Serous cystadenoma. This has a very low potential for malignant transformation (rarely being associated with cystadenocarcinoma), suggesting a management determined by symptoms. Serous cystadenomas can be managed by observation, since the risk of malignant changes is small [8]. This lesion is typically composed of a myriad of microcysts, $2 \mathrm{~mm}$ or less in size, which sometimes appear "layered" during EUS examination [6]. These small cysts are often associated with macrocysts of varying sizes, with thin walls and no vascular enhancement on CT scanning. In less than $30 \%$ of cases, a pathognomonic central calcified scar is identified [4,7]. In $10 \%$ of cases, the serous cystadenoma is composed of a unique macrocyst. In this particular case, the differential diagnosis from mucinous cystadenoma is subtle and EUS fine-needle aspiration is useful in order to establish the final diagnosis. The cell content of the cystic liquid of serous cystadenomas is often poor (25\%), while it is rich in the case of mucinous cystadenomas (30\%-75\%) [2]. Normal amylase and low levels of tumor markers (i.e. CEA and CA 19-9) in the cystic liquid are typical of a serous cystadenoma [6]. Nevertheless, an increased concentration of CA 19-9 may sometimes be encountered in a serous cystadenoma or a pancreatic pseudocyst. However, a CA 19-9 level greater than $50000 \mathrm{IU} / \mathrm{ml}$ is suggestive of mucinous cystadenoma $[2,6]$. The clinician must bear in mind that the morbidity of the cystic puncture is not zero, being $1 \%$ to $4 \%$. This morbidity is mainly accounted for by infection, which justifies systematic antibiotic prophylaxis $[2,6]$. Measurement of the CA $72-4$ level is not relevant as there is an important overlap.

\section{Management}

$\nabla$

When there is a definitive diagnosis of serous cystadenoma, the patient does not need a specific follow-up. An ultrasound checkup or a CT scan 1 year after the diagnosis has been established is recommended, as these lesions can enlarge and become sympto- 
matic as a result of displacement or compression of nearby organs [8].

Mucinous cystadenomas and solid pseudopapillary tumors must be resected surgically $[2,7]$.

The management of IPMT is still a subject of controversy. When the main pancreatic duct is affected by the disease, as indicated by tumor ingrowth or dilatation of the main pancreatic duct by more than $10 \mathrm{~mm}$, a surgical resection is indicated $[9,10]$. The extent of the pancreatic resection is based on preoperative staging that could include a pancreatoscopy or an intraductal miniprobe EUS examination, and also on the examination of frozen sections of the resection margins during surgery $[2,10]$. When the disease only affects branch ducts, the lesion can be kept under observation if the patient is asymptomatic, and on condition that the main lesion measures less than $30 \mathrm{~mm}$ and there are no endocystic polyps or nodules [10]. However, the surgical risk of a pancreatic resection must be weighed against the possible risk of subsequent malignancy, taking into account the age of the patient and the size of the pancreatic resection. Segmental resection of the tumor, or enucleation, may be considered if the lesion is localized.

With regard to conservative management, modalities and recommendations for follow-up are not clearly established in the literature. The standard is yearly EUS, as it is the most accurate imaging technique in this particular case. A more strict schedule is to alternate EUS and CT scan every 6 months. If the patient has an elevated sedation risk, yearly ultrasonography (if the lesion is visible) or CT scan is considered acceptable. Also, if the lesion remains unchanged over 2 years, the follow-up interval may be extended to 2 years.

Competing interests: None

\section{References}

1 Ikeda M, Sato T, Morozumi A et al. Morphologic changes in the pancreas detected by screening ultrasonography in a mass survey, with special reference to main duct dilatation, cyst formation and calcification. Pancreas 1994; 9: 508 - 512

2 Hammel $P$. Tumeurs pancréatiques de découverte fortuite: diagnostic et prise en charge. Gastroenterol Clin Biol 2002; 26: 700-708

3 Brugge WR, Lewandrowski K, Lee Lewanfrowski $E$ et al. Diagnosis of pancreatic cystic neoplasms: a report of the cooperative pancreatic cyst study. Gastroenterology 2004; 126: 1330-1336

4 Warshaw AL, Compton C, Lewandrowski K et al. Cystic tumors of the pancreas. New clinical, radiological and pathological observations in 67 patients. Ann Surg 1990; 212: 432 - 443

5 Cohen-Scali F, Vilgrain V, Brancatelli G et al. Discrimination of unilocular macrocystic serous cystadenoma from pancreatic pseudocyst and mucinous cystadenoma with CT: initial observations. Radiology 2003; 228: 727-733

6 O'Toole D, Palazzo L, Hammel P et al. Macrocystic pancreatic cystadenoma. The role of EUS and cyst fluid analysis in distinguishing mucinous and serous lesions. Gastrointest Endosc 2004; 59: 823 - 829

7 Le Borgne J, De Calan L, Partensky C et al. Cystadenoma and cystadenocarcinomas of the pancreas: a multiinstitutional retrospective study of 398 cases. French Surgical Association. Ann Surg 1999; 230: $152-$ 161

8 Scheiman JM. Cystic lesions of the pancreas. Gastroenterology 2005; 128: $463-469$

9 Okabayashi T, Kobayashi M, Nishimori I et al. Clinicopathological features and medical management of intrapancreatic mucinous neoplasms. J Gastroenterol Hepatol 2006; 21: 462 - 467

10 Matsumoto T, Aramaki M, Yada $K$ et al. Optimal management of the branch duct type intraductal papillary mucinous neoplasms of the pancreas. J Clin Gastroenterol 2003; 36: 261 - 265 